\title{
TOPOLOGICAL TYPES OF POLYNOMIAL DIFFERENTIAL EQUATIONS
}

\author{
BY
}

\author{
LAWRENCE MARKUS( $\left.{ }^{1}\right)$
}

\begin{abstract}
Consider a first order system of real ordinary differential equations, with polynomial coefficients, having no critical points in the number space $R^{n}$. Two such differential systems are called topologically equivalent in case there exists a homeomorphism of $R^{n}$ onto itself carrying the sensed (not parametrized) solutions of the first system onto the solution family of the second system. Let $B^{n}(m)$ be the cardinal number of topological equivalence classes for systems in $R^{n}$ with polynomial coefficients of degree at most $m$. The author proves that $B^{2}(m)$ is finite and obtains explicit upper and lower bounds in terms of $m$. Also examples are given to show that $B^{n}(m)$ is noncountable for $n \geq 3$ and $m \geq 6$.
\end{abstract}

1. Topological equivalence of differential systems. Examples. Ordinary differential equations will be considered to be topologically equivalent in case their solution curve families are homeomorphic. We shall examine differential equations having polynomial coefficients of degree at most $m$, without critical points in the real plane, and we shall explicitly estimate the finite number $B(m)$ of topological types of such equations. Examples show that replacing the plane by 3-space, or the polynomials by real analytic functions, or the homeomorphisms by diffeomorphisms, leads to unreasonable problems with a continuum of equivalence classes of differential systems.

Consider an autonomous differential system

$$
\frac{d x^{i}}{d t}=\dot{x}^{i}=f^{i}\left(x^{1}, x^{2}, \cdots, x^{n}\right), \quad i=1,2, \cdots, n,
$$

with real coefficients $f(x)=\left(f^{i}\left(x^{1}, x^{2}, \cdots, x^{n}\right)\right)$ continuously differentiable, that is, in class $C^{1}$, in the real number $n$-space $R^{n}$. For each initial point $x_{0}=\left(x_{0}^{1}, x_{0}^{2}, \cdots, x_{0}^{n}\right)$ in $R^{n}$, there is a unique solution curve $x\left(t, x_{0}\right)$ initiating at $x\left(0, x_{0}\right)=x_{0}$ and defined on some maximal time duration. We shall be concerned only with the sensed, rather than the time-parametrized curves. That is, each parametrized solution curve $x\left(t, x_{0}\right)$ determines the sensed solution which consists of the class of all curves $x\left(\tau(t), x_{0}\right)$, where $\tau(t)$ is any continuous and strictly

Received by the editors March 29, $197 \mathrm{i}$ and, in revised form, November 8, 1971. AMS 1970 subject classifications. Primary 34C35; Secondary 14D05.

Key words and phrases. Polynomial differential equations, foliation, topological equivalence, separatrix, orbit space.

(1) This research was partially supported by ONR contract No. 00014-67-A-0113-0019 and represents the completion of the ideas of the author's paper [6] submitted to this journal twenty years ago. The first version of this paper was transmitted to the editors and returned for author modifications ten years ago. 
monotonic increasing change of parameter. Thus for sensed solutions we can always assume the solutions to be defined on all $-\infty<t<\infty$. We shall study the topological structure of the family of all sensed solution curves of the differential system $\mathcal{S}$ in $R^{n}$.

Definition. The differential systems

$\left(\mathcal{S}_{1}\right) \quad \dot{x}=f_{1}(x)$,

$\left(\mathcal{S}_{2}\right) \quad \dot{x}=f_{2}(x)$,

with the real vector functions $f_{1}(x)$ and $f_{2}(x)$ in $C^{1}$ in $R^{n}$, are topologically equivalent in case there exists a homeomorphism $\Phi$ of $R^{n}$ onto itself carrying the sensed solution curve family of $\mathcal{S}_{1}$ onto that of $\mathfrak{S}_{2}$.

Remarks. The terminology of the definition means that $\Phi$ carries each sensed solution curve of $\mathcal{S}_{1}$ onto an entire sensed solution of $\mathcal{S}_{2}$, and vice versa for $\Phi^{-1}$. Under the correspondence induced by $\Phi$, the critical points (equilibrium, stationary, or singular points) of $\mathcal{S}_{1}$ correspond to critical points of $\mathcal{S}_{2}$; the periodic orbits (closed orbits, periodic solutions) of $\mathcal{S}_{1}$ correspond to periodic orbits of $\mathcal{S}_{2}$. Also invariant sets and minimal sets of $\mathcal{S}_{1}$ and $\mathcal{S}_{2}$ must correspond. In the plane $R^{2}$ each solution of a noncritical differential system $\mathcal{S}$ is a topological image of a line, extending to infinity along both ends, and separating the plane into exactly two regions. It is this separation phenomenon that makes such differential systems in $R^{2}$ simple enough for description and topological classification, as explained later.

Definition. Consider the set $\mathcal{P}^{n}(m)$ of all differential systems

$$
\dot{x}=f(x) \text {. }
$$

with each component of $f(x)$ a polynomial of degree at most $m$, and with no critical points in $R^{n}$. We denote by $B^{n}(m)$ the cardinality of the set of all topological equivalence classes in $\mathscr{P}^{n}(m)$.

Remark. If we consider differential systems $\mathcal{S}$ as above, without critical points in $R^{n}$, and if we ignore the sense as well as the parametrization of the solutions, then the solution curves define a regular curve family ([4], [9]) or foliation of $R^{n}$. We define the topological equivalence of two such foliations of $R^{n}$, as specified by differential systems $\mathcal{S}_{1}$ and $\mathcal{S}_{2}$ by means of a homeomorphism of $R^{n}$ on to itself that carries each unsensed solution of $\mathcal{S}_{1}$ onto an unsensed solution of $\mathcal{S}_{2}$.

Just as above we let $B_{f}^{n}(m)$ be the cardinality of the set of all topological equivalence classes of foliations arising from polynomial differential systems of $\rho^{n}(m)$.

We shall also define the equivalence relation of topological 0-equivalence on the polynomial differential systems of $\mathcal{P}^{n}(m)$, and on the foliations that they determine, by the further requirement that the homeomorphism $\Phi$ specifying the 
topological equivalence must preserve the orientation of $R^{n}$. We denote the cardinality of the set of topological 0-equivalence classes of $\mathcal{P}^{n}(m)$ by $B_{0}^{n}(m)$, and the corresponding number of 0 -equivalence classes of foliations by $B_{f 0}^{n}(m)$.

Each topological equivalence class of foliations specified by $\mathcal{P}^{n}(m)$ contains two topological equivalence classes of differential systems (one for each possible choice of sense), except when these two classes happen to coalesce. Thus $B_{f}^{n}(m) \leq B^{n}(m) \leq 2 B_{f}^{n}(m)$, and a similar consideration for 0 -equivalence classes yields $B_{f 0}^{n}(m) \leq B_{0}^{n}(m) \leq 2 B_{f 0}^{n}(m)$. Also, since $R^{n}$ has just two orientations, $B_{f}^{n}(m) \leq B_{f 0}^{n}(m) \leq 2 B_{f}^{n}(m)$. From these inequalities we conclude that

$$
B^{n}(m) \leq 2 B_{f 0}^{n}(m) \leq 4 B^{n}(m),
$$

and so it is possible to estimate $B^{n}(m)$ from bounds for $B_{f 0}^{n}(m)$. We shall show that $B^{n}(m)$ is the continuum $\boldsymbol{K}_{1}$ for $n \geq 3$ and $m \geq 6$ by examples presented below. In the next section we shall prove that $B^{2}(m)$ is finite, and we shall give upper and lower bounds depending on the integer $m$. In particular, we obtain the asymptotic behavior as $m \rightarrow \infty$,

$$
\log \log B^{2}(m) \sim \log m .
$$

Example 1. We first show that $B^{n}(0)=B^{n}(1)=1$. That is, for linear differential systems without critical points in $R^{n}$, there is just a single topological equivalence class.

Consider a differential system in $\mathfrak{P}^{n}(1)$, written in matrix notation,

$$
\dot{x}=A x+b \text {. }
$$

Since $\mathcal{S}$ has no critical points, the vector $b \neq 0$ and $b$ does not lie in the range of the linear transformation specified by the matrix $A$. Since a nonsingular linear transformation of $R^{n}$ carries $\mathcal{S}$ into a polynomial differential equation of the same degree, we shall assume that the basis for $R^{n}$ has already been chosen to consist of the vector

$$
b=\left(\begin{array}{c}
1 \\
0 \\
0 \\
\vdots \\
0
\end{array}\right),
$$

and vectors $e_{2}, e_{3}, \cdots, e_{n}$, which span the range of $A$. Then the first row of $A$ is zero, and $\mathcal{S}$ has the form

$$
\dot{x}^{1}=1,
$$$$
\dot{x}^{i}=\sum_{j=1}^{n} a_{j}^{i} x^{j} \quad \text { for } i=2,3, \cdots, n \text {. }
$$ 
Because $\dot{x}^{1}=1$, each solution curve of $\mathcal{S}$ meets the hyperplane $x^{1}=0$ in exactly one point $x_{0}$ and can thus be written $x\left(t, x_{0}^{2}, x_{0}^{3}, \cdots, x_{0}^{n}\right)$. Now we can map $\mathcal{S}$ (even differentiably) onto the parallel system

$$
\begin{aligned}
& \dot{u}^{1}=1, \\
& \dot{u}^{i}=0 \text { for } i=1,2, \cdots, n,
\end{aligned}
$$

by using curvilinear coordinates $u=u(x)$ in $R^{n}$. We define $u(x)$ by means of the transversal $x^{1}=0$ and the solution curves $x\left(t, x_{0}^{2}, \cdots, x_{0}^{n}\right)$. For each point $\bar{x} \in R^{n}$, let $x\left(\bar{t}, \bar{x}_{0}^{2}, \cdots, \bar{x}_{0}^{n}\right)=\bar{x}$, and define $u(\bar{x})=\left(\bar{t}, \bar{x}_{0}^{2}, \cdots, \bar{x}_{0}^{n}\right)$. Thus $\mathcal{S}$ is topologically equivalent to the standard parallel system in $R^{n}$, and so $B^{n}(1)=1$.

Example 2. We examine differential systems of $\mathcal{P}^{2}(m)$ and obtain a lower bound for $B^{2}(m)$, in particular $B^{2}(m) \geq 2^{m / 2}$ for $m \geq 36$. In subsequent remarks, using the same techniques developed in this example, we point out that $\mathscr{P}^{2}(\infty)$, the set of real analytic differential systems without critical points in the plane, has the cardinality $\boldsymbol{N}_{1}$ of the continuum for the number $B^{2}(\infty)$ of topological types.

Consider the differential system in the $(x, y)$-plane $R^{2}$

$$
\begin{aligned}
& \dot{x}=x(x-1)(x-2) \cdots(x-k), \\
& \dot{y}=(x-1 / 2)(x-3 / 2) \cdots(x-k+1 / 2),
\end{aligned}
$$

for integers $k \geq 1$. The vertical lines $x=0, x=1, \cdots, x=k$ are each solution curves and they bound $k$ strips or regions between them. Further the sense along the solutions $x=0, x=1, \cdots, x=k$ is alternately upwards and downwards (upwards on $x=k$ ). This forces each of the strips to be filled with $U$-shaped solutions concave downwards.

We next note that this description of the solution curve family utilizes topological invariants. Each of the solutions $x=0, x=1, \cdots, x=k$ is a separatrix in the following sense: in the quotient or identification topology on the set of solutions each of these $k+1$ line solutions defines an element that is inseparable by open sets from some other element. That is, the solutions $x=0, x=1, \cdots$, $x=k$ are the non-Hausdorff elements of the orbit or solution quotient space (see technical definitions in $\$ 2$ of this paper). Clearly, these $k+1$ lines are the only separatrices of the differential system, since any other solution curve $S$ has neighboring solutions $S_{1}$ and $S_{2}$ such that $S_{1}$ and $S_{2}$ are the boundary of a topological closed strip $\left[S_{1} S_{2}\right]$ that contains $S$ and contains no cyclic triples of solutions (three distinct solutions are always placed such that either one separates the other two in the plane, or no such separation arises in which case we say that the three curves form a cyclic triple-see $\$ 2$ below).

The number of separatrices of a differential system is a topological invariant of the system. The above differential system has $k+1$ separatrices and belongs to $\mathcal{P}^{2}(k+1)$. By omitting some of the factors in the expressions for $\dot{x}$ and $\dot{y}$, we can construct differential systems in $\mathcal{P}^{2}(\mathrm{~m})$ with any number of separatrices from 
2 to $m=k+1$. Therefore, $B^{2}(m) \geq m-1$ for $m \geq 2$.

Of course, $B^{2}(2) \geq 2$ since we could fill the single strip region between $x=0$ and $x=1$ by solutions parallel to the boundary solutions to obtain another topological equivalence class of $\mathfrak{P}^{2}(2)$. We exploit this device to find a greater lower bound for $B^{2}(m)$.

Consider the differential systems in $R^{2}$,

$$
\begin{aligned}
& \dot{x}=x(x-1)^{a_{1}}(x-2)^{a_{2}} \cdots(x-k+2)^{a_{k-2}}(x-k+1)(x-k)^{\alpha_{k}}, \\
& \dot{y}=(x-1 / 2)(x-3 / 2) \cdots(x-k+5 / 2)(x-k+1 / 2),
\end{aligned}
$$

for integers $k \geq 3$, and integers $\alpha_{j}=1$ or 2 . We shall show that these differential systems, for various choices of $\alpha_{j}$, define at least $2^{k-1} / 4$ topological types for systems in $\mathfrak{P}^{2}(2 k)$.

The vertical lines $x=0, x=1, x=2, \cdots, x=k-1, x=k$ are each solution curves and bound $k$ strips or regions between them. Also the sense of the solutions $x=0, x=1, x=2, \cdots, x=k-2$ is alternately upwards and downwards, but the last three lines at $x=k-2, x=k-1, x=k$ are always down, down, up, respectively. This forces each of the first $k-3$ regions to be filled with $U$ shaped solutions that are concave upwards or downwards depending on the sign of $\dot{x}$ in that strip, that is, depending on the choices for $a_{j}=1$ or 2 . Thus the total picture consists of $k+1$ solutions that bound $k$ strip regions, the first $k-3$ regions filled in a cyclic pattern (cyclic with the two boundary curves), and the last three regions being distinctive with two cyclic regions on either side of a region containing no cyclic set of solutions (even in the closure of the region).

Again we note that the above description of the solution curve family is essentially topological. Each of the solutions $x=0, x=1, \cdots, x=k$ is a separatrix, and these are the only separatrices. Thus the $k+1$ separatrices are topologically distinguished together with the $k+2$ regions, called the canonical regions, that they bound in the plane. Furthermore, the $k-1$ canonical regions, that each contain a cyclic triple of curves in the closed region, are also topologically distinguished.

Now fix $k \geq 4$ and consider two differential systems $\mathcal{S}_{\alpha}$ and $\delta_{\bar{a}}$ for two choices of the exponent arrays $a=\left(a_{1}, \alpha_{2}, \ldots, \alpha_{k-2}, a_{k}\right)$ and $\bar{\alpha}=\left(\bar{a}_{1}, \bar{\alpha}_{2}, \ldots, \bar{\alpha}_{k-2}, \bar{\alpha}_{k}\right)$. If $\mathcal{S}_{a}$ is topologocially equivalent to $\mathcal{S}_{\bar{a}}$, then the $k+1$ separatrices of both must correspond, and in the obvious linear order (or its reverse) because of plane separation considerations. Let us assume a direct correspondence of the separatrices of $\mathcal{S}_{a}$ and $\mathcal{S}_{\bar{a}}$, which is necessarily the case when $k \geq 4$ since the last two strip canonical regions are distinctive. We also assume the topological map $\Phi$ carrying $\mathcal{S}_{\boldsymbol{\alpha}}$ onto $\mathcal{S}_{\bar{a}}$ preserves the orientation of $R^{2}$, so positive or counterclockwise cyclic triples of solutions of $\varsigma_{a}$ are carried to positive cyclic triples of $\delta_{a}$. Now note that if $a_{k}=1$ so $\dot{x}$ in $\delta_{a}$ changes sign at $x=k$, then the three curves, $x=k$, 
$x=k-1$, and any solution from the canonical region these two separatrices bound, form a positive cyclic triple. If $\alpha_{k}=2$ so $\dot{x}$ in $\mathcal{S}_{a}$ does not change sign at $x=k$, then the corresponding cyclic triple is clockwise or negative.

Since we suppose that $\mathcal{S}_{a}$ and $\mathcal{S}_{\bar{a}}$ are topologically equivalent under an orientation-preserving homeomorphism $\Phi$, we conclude that $\alpha_{k}=\bar{\alpha}_{k}$. In the same way we prove that $\alpha_{j}=\bar{\alpha}_{j}$ for $j=2,3, \cdots, k-2$.

Since there are $2^{k-1}$ choices of the exponents $\alpha$, we have constructed $2^{k-1}$ different systems all of degree at most $m=2(k-1)+2=2 k$. Of course, if we allow $\Phi$ to interchange the order of the separatrix lines in $\delta_{a}$, or reverse orientation of $R^{2}$, we can be assured of only $2^{k-1} / 4$ topologically distinct classes in $\mathcal{P}^{2}(2 k)$. But the distinguished strip between $x=k-1$ and $x=k$ could be placed instead in any one of the $k-2$ strips intermediate between the first and last strip. Thus we construct $(k-2) 2^{k-1} / 4$ topologically distinct systems of $\mathcal{P}^{2}(2 k)$. In fact, since we have ignored the sense along the solutions of $\mathcal{S}$ when considering the map $\Phi$, we have proved that

$$
B_{f}^{2}(m) \geq[(m-4) / 8] 2^{m / 2-1} \text { for } m=2 k \geq 8 .
$$

For $m \geq 36$ we compute $(m-4) / 8 \geq 4$ so $B_{f}^{2}(m) \geq 2^{m / 2+1}$. For odd values of $m \geq 37$ we can use

$$
B_{f}^{2}(m) \geq B_{f}^{2}(m-1) \geq 2^{(m-1) / 2+1}=2^{(m+1) / 2} \geq 2^{m / 2} .
$$

Thus in every case

$$
B^{2}(m) \geq B_{f}^{2}(m) \geq 2^{m / 2} \quad \text { for } m \geq 36 .
$$

Note. The lower bound $B^{2}(m) \geq 2^{m / 2}$, for large $m$, is a significant result that will be used later.

Remark. Consider the set $\mathscr{P}^{2}(\infty)$ of real analytic differential systems without critical points in $R^{2}$. We shall indicate a continuum of topologically distinct systems in $\mathscr{P}^{2}(\infty)$. Since the cardinality of $\mathcal{P}^{2}(\infty)$ is the continuum $\boldsymbol{N}_{1}$, it will follow that $B^{2}(\infty)=\boldsymbol{K}_{1}$. Consider the differential systems

$$
\begin{aligned}
& \dot{x}=E(x) x(x+1)^{\alpha}(x+2)^{\alpha} 2 \ldots(x+k)^{\alpha} \ldots, \\
& \dot{y}=E(x)(x+1 / 2)(x+3 / 2)(x+5 / 2) \cdots,
\end{aligned}
$$

where $E(x)$ is a suitable exponential to insure that the infinite products converge to entire functions. The parameter $a=\left(\alpha_{1}, \alpha_{2}, \cdots\right)$ is a sequence of $\alpha_{k}=1$ or 2 .

The only separatrices of $\mathcal{S}_{a}$ are $x=0, x=-1, x=-2, \ldots, x=-k, \cdots$. These separatrices bound a countable number of canonical regions, which contain $U$-solutions opening upwards or downwards according to the sign of $\dot{x}$, or the value of $a$. Using the arguments of the above example we can prove that $\mathcal{S}_{a}$ is topologically equivalent to $\mathcal{S}_{\bar{a}}$ if and only if $a=\bar{\alpha}$. Since the set of sequences 
$\alpha=\left(\alpha_{1}, \alpha_{2}, \cdots\right)$ has the cardinality of the continuum, we conclude that $B^{2}(\infty)=\boldsymbol{K}_{1}$.

Example 3. In this example we illustrate the futility of trying to classify differential systems in $\mathcal{P}^{2}(m)$ under the relation of differential equivalence (where the homeomorphism $\Phi$ is a $C^{1}$-diffeomorphism of $R^{2}$ onto itself). Our example displays a continuum of such equivalence classes for $m \geq 3$.

Consider the differential systems $\delta_{a}$ in $\mathscr{P}^{2}(3)$ in the $(x, y)$-plane,

$$
\dot{x}=x(x-2)(1+\alpha x), \quad \dot{y}=(x-1),
$$

where $\alpha>0$ is a real parameter.

The two lines $x=0$ and $x=2$ are the only separatrices, since $\dot{y}$ does not change sign for $x \geq 2$ or for $x \leq 0$. Between these separatrices the solutions are $U$-shaped opening downwards, and given by

$$
\int_{y_{0}}^{y} d y=\int_{x_{0}}^{x} \frac{(x-1) d x}{x(x-2)(1+\alpha x)} .
$$

We take the initial point $x_{0}=2-\xi, y_{0}=0$ for small $\xi>0$, and compute the reintercept $x(\xi)$ on the $x$-axis by

$$
\int_{2-\xi}^{x(\xi)} \frac{(x-1) d x}{x(x-2)(1+\alpha x)} \equiv 0
$$

From the geometric viewpoint we have chosen two segments of the $x$-axis as transversals through the separatrices, and then we have studied the map between these transversals as defined by the solution curves.

The map $\xi \rightarrow x(\xi)$ for $\xi \searrow 0$ satisfies

$$
\frac{(x-1)}{x(x-2)(1+\alpha x)} \frac{d x}{d \xi}-\left[\frac{(1-\xi)}{(2-\xi)(-\xi)(1+2 \alpha-\alpha \xi)}\right](-1)=0
$$

or

$$
\frac{d x}{x} / \frac{d \xi}{\xi}=\frac{(1-\xi)}{(2-\xi)(1+2 \alpha-\alpha \xi)} \frac{(x-2)(1+\alpha x)}{(x-1)} .
$$

Therefore, along the map $\xi \rightarrow x(\xi)$ as $\xi \searrow 0$,

$$
\frac{d \log x}{d \log \xi} \rightarrow \frac{1}{1+2 \alpha}
$$

If we select different $C^{1}$-transversals across the two separatrices, say,

$$
\begin{aligned}
& x=x_{1}(u), \quad y=y_{1}(u) \quad \text { with } x_{1}(0)=0, x_{1}^{\prime}(0)>0, \\
& x=x_{2}(\eta), \quad y=y_{2}(\eta) \quad \text { with } x_{2}(0)=2, x_{2}^{\prime}(0)<0,
\end{aligned}
$$

then we define the map $\eta \rightarrow u(\eta)$ by the solutions

$$
\int_{y_{2}(\eta)}^{y_{1}(u(\eta))} d y=\int_{x_{2}(\eta)}^{x_{1}(u(\eta))} \frac{\left(x_{0}-1\right) d x}{x(x-2)(1+\alpha x)} .
$$


Hence

$$
\frac{d y_{1}}{d u} \frac{d u}{d \eta}-\frac{d y_{2}}{d \eta}=\frac{\left(x_{1}-1\right)}{x_{1}\left(x_{1}-2\right)\left(1+\alpha x_{1}\right)} \frac{d x_{1}}{d u} \frac{d u}{d \eta}-\frac{\left(x_{2}-1\right)}{x_{2}\left(x_{2}-2\right)\left(1+\alpha x_{2}\right)} \frac{d x_{2}}{d \eta}
$$

and

$$
\frac{d \log u}{d \log \eta}=\left[\frac{\left(x_{2}-1\right) x_{2}^{\prime}}{x_{2}\left(1+\alpha x_{2}\right)}-y_{2}^{\prime}\left(x_{2}-2\right)\right]\left[\frac{\left(x_{1}-1\right) x_{1}^{\prime}}{\left(x_{1}-2\right)\left(1+\alpha x_{1}\right)}-y_{1}^{\prime} x_{1}\right]^{-1} \frac{\eta}{u} \frac{x_{1}}{x_{2}-2} \text {. }
$$

Now as $\eta \searrow 0, x_{1} \rightarrow 0$ and $x_{2} \rightarrow 2$ so

$$
\frac{d \log u}{d \log \eta} \rightarrow \frac{x_{2}^{\prime}(0)}{x_{1}^{\prime}(0)} \frac{1}{1+2 \alpha}\left[\lim _{\eta \rightarrow 0} \frac{\eta}{u} \frac{x_{1}}{x_{2}-2}\right]=\frac{1}{1+2 \alpha}
$$

Thus the number $1 /(1+2 \alpha)$ is an invariant of the differential system $\mathcal{S}_{a}$, and is preserved under any $C^{1}$-diffeomorphism of this system. Hence for a change in the real parameter $\alpha$, we change the differential equivalence class of $\mathcal{S}_{a}$. Therefore there exist a noncountable continuum of such equivalence classes among the differential systems $\mathcal{S}_{\alpha}$.

By slight modifications we construct a continuum of differential equivalence classes for noncritical polynomial differential systems of degree $m \geq 3$, but we omit the details.

Example 4. In this example we illustrate the futility of trying to classify differential systems in $\mathcal{P}^{n}(m)$ for $n \geq 3$, even under the usual relation of topological equivalence. Our example displays a continuum of equivalence classes, so $B^{n}(m)=$ $K_{1}$ for all $n \geq 3, m \geq 6$.

Consider the differential system $\mathcal{S}_{\lambda}$ in $\mathcal{P}^{3}(6)$ in the $(x, y, z)$-space $R^{3}$,

$$
\begin{aligned}
& \dot{x}=-1 / 2 x z\left(x^{2}+y^{2}\right)^{2}-\lambda y, \\
& \dot{y}=-1 / 2 y z\left(x^{2}+y^{2}\right)^{2}+\lambda x, \\
& \dot{z}=-\left(1-2 x^{2}-2 y^{2}\right),
\end{aligned}
$$

where $\lambda$ is an irrational real parameter. Note that the line $x=y=0$ is invariant and therefore $\dot{z}=-1$. Outside of the $z$-axis, we use cylindrical coordinates $\rho^{2}=$ $x^{2}+y^{2}, \theta=\arctan (y / x), z$ to write the differential system $\delta_{\lambda}$ in the form $\dot{\rho}=$ $-1 / 2 z \rho^{5}, \dot{\theta}=\lambda, \dot{z}=-\left(1-2 \rho^{2}\right)$. Since $\dot{\theta} \neq 0$, we see that $\mathcal{J}_{\lambda}$ has no critical point in $R^{3}$, and hence it defines a polynomial system in $\mathcal{P}^{3}(6)$ in the $(x, y, z)$ space.

Next introduce the new coordinate $u=1 / \rho^{2}>0$ to write $\delta_{\lambda}$ in a form where the qualitative nature of the solutions is evident, $\dot{u}=z / u, \dot{z}=-(u-2) / u, \dot{\theta}=\lambda$.

With respect to the $(u, z)$-coordinates the motion is like that of a linear oscillator about $u=2, z=0$, except that the factor $1 / u$ causes a singularity at $u=0$ 
(where $\rho=\infty)$. The point $u=2, z=0$ is a fixed center about which the $(u, z)$ flow moves in circles $(u-2)^{2}+z^{2}=r^{2}$, for $0<r<2$, with some period $T(r)$ which is continuous and approaches $4 \pi$ as $r \rightarrow 0$.

In the $(x, y, z)$-space $R^{3}$ the point $u=2, z=0$ determines a periodic orbit $S_{0}$ with period $2 \pi / \lambda$, and each of the above circles determines an invariant torus surface $T_{r}$ of the system $\mathcal{S}_{\lambda}$. The torus $T_{r}$ is filled by periodic orbits of $\mathcal{S}_{\lambda}$ if and only if $T(r)$ and $2 \pi / \lambda$ are rationally dependent, otherwise $T_{r}$ is a minimal set, since then every solution curve in $T_{r}$ is dense in $T_{r}$. Thus the only compact minimal sets for $\mathcal{S}_{\lambda}$ in $R^{3}$ are periodic orbits, such as $S_{0}$, and the periodic orbits filling certain of the tori $T_{r}$, and certain two-dimensional torus surfaces corresponding to $T_{r}$ for which $2 \pi /(\lambda T(r))$ is irrational.

We now proceed to show that $\lambda$ determines a topological invariant of $\mathcal{S}_{\lambda}$ in $R^{3}$. First we characterize the periodic orbit $S_{0}$ topologically. If the period $T(r)$ is identically $4 \pi$ for all small $r>0$ then there exist arbitrarily narrow minimal tori $T_{r}$ enclosing the periodic orbit $S_{0}$ in their interior region in $R^{3}$. If $T(r)$ is not constant for all small $r>0$, then for certain arbitrarily small $r_{1}>0$ we have $2 \pi /\left(\lambda T\left(r_{1}\right)\right)$ irrational, and so there are arbitrarily narrow minimal tori enclosing $S_{0}$. Thus we recognize $S_{0}$ as the unique periodic orbit of $\delta_{\lambda}$ in $R^{3}$ that lies inside every minimal torus.

Take any minimal torus $T_{r_{1}}$ of $\mathcal{S}_{\lambda}$ and consider the solid torus which it bounds in $R^{3}$. Let $A$ denote this solid torus with the curve $S_{0}$ deleted. Then $T_{r}$ is a deformation retract of $A$, and so we can pick two 1 -cycles in $A$ to specify a basis for the integral, singular homology group $H_{1}(A)=\mathbf{Z} \times \mathbf{Z}$. The injection map of $T_{r_{1}}$ into $A$ induces a canonical isomorphism of the 1-homology group of $T_{r_{1}}$ onto $H_{1}(A)$.

Choose a point $P_{0} \in T_{r_{1}}$ and let $P_{t}$ for $t \geq 0$ be the solution curve of $\mathcal{S}_{\lambda}$ initiating at $P_{0}$. Choose a disc neighborhood $N$ of $P_{0}$ in $T_{r_{1}}$ and note that $P_{t}$ recurs to the neighborhood $N$ for arbitrarily large times $t_{k} \rightarrow \infty$. Choose a curve $l_{k} \subset N$ joining $P_{t_{k}}$ to $P_{0}^{\prime}$ and consider the closed curve $C_{k}$ consisting of the solution arc $P_{0} P_{t_{k}}$ followed by the curve $l_{k}$ joining $P_{t_{k}}$ back to $P_{0}$. Then $C_{k}$ determines a homology class $H_{1}(A)$, which is a pair of integers $\left(\alpha_{k}, \beta_{k}\right)$ in terms of the given basis.

From the general theory of differential equations on a torus [8], it is known that

$$
\lim _{k \rightarrow \infty} \frac{\beta_{k}}{a_{k}}=\mu,
$$

which is the classical rotation number for $\mathcal{S}_{\lambda}$ on $T_{r_{1}}$. Since $\mathcal{S}_{\lambda}$ is minimal on $T_{r_{1}}$ we know that $\mu$ is irrational.

Now select a sequence of such minimal tori $T_{r_{j}}$ lying in $A$ and tending towards $S_{0}$. Let corresponding rotation number of $\mathcal{S}_{\lambda}{ }^{j}$ on $T_{r_{j}}$ be $\mu\left(r_{j}\right)$ (with respect 
to the fixed homology basis of $\left.H_{1}(A)\right)$. First take the homology basis of $H_{1}(A)$ given by the image of the cycles $u=2+r_{0}, z=0,0<\theta \leq 2 \pi$ and $(u-2)^{2}+z^{2}=r_{0}^{2}$, $\theta=0$, around which the time periods of $\mathcal{S}_{\lambda}$ are approximately $2 \pi / \lambda$ and $4 \pi$ (for some suitably small $r_{0}>0$ ). Thus $\alpha_{k} \cdot 2 \pi / \lambda$ is nearly equal to $\beta_{k} \cdot 4 \pi$ and so

$$
\lim _{j \rightarrow \infty} \mu\left(r_{j}\right)=\lim _{j \rightarrow \infty}\left[\lim _{k \rightarrow \infty} \frac{\beta_{k}}{\alpha_{k}}\right]=\frac{1}{2 \lambda} .
$$

This computation of $1 / 2 \lambda$ depends only on the topology of the differential system $\mathcal{S}_{\lambda}$ and the choice of the integral homology base for $H_{1}(A)$.

If another integral homology base for $H_{1}(A)$ is chosen, it is related to the above base by an automorphism of $\mathbf{Z} \times \mathbf{Z}$ determined by a unimodular matrix $\left(\begin{array}{ll}a & b \\ c & d\end{array}\right)$. The corresponding rotation number of $\delta_{\lambda}$ on $T_{r_{j}}$ is $\left(c+d \mu\left(r_{j}\right)\right) /\left(a+b \mu\left(r_{j}\right)\right)$, which gives the limiting value

$$
\lim _{j \rightarrow \infty} \frac{c+d \mu\left(r_{j}\right)}{a+b \mu\left(r_{j}\right)}=\frac{2 \lambda c+d}{2 \lambda a+b} .
$$

We define two irrational numbers $\mu_{1}$ and $\mu_{2}$ to be equivalent in case there exists a unimodular transformation $\left(\begin{array}{ll}a & b \\ c & d\end{array}\right)$ such that $\mu_{2}=\left(c+d \mu_{1}\right) /\left(a+b \mu_{1}\right)$. Since there are only a countable number of unimodular matrices, there are a continuum $\boldsymbol{N}_{1}$ of equivalence classes of irrational numbers (incidentally, $\mu_{1}$ and $\mu_{2}$ are equivalent if and only if their simple continued fraction expansions coincide after a finite number of initial digits are discarded from each [3]):

Thus the parameter $\lambda$ in $\mathcal{S}_{\lambda}$ specifies an equivalence class of irrational numbers, and this class of irrationals is a topological invariant of the differential system $\mathcal{S}_{\lambda}$. If $1 / 2 \lambda_{1}$ is not equivalent to $1 / 2 \lambda_{2}$, then $\mathcal{S}_{\lambda_{1}}$ is topologically distinct from $\mathcal{S}_{\lambda_{2}}$. Thus $B^{3}(6)=\boldsymbol{N}_{1}$ and so $B^{3}(m)=\boldsymbol{\aleph}_{1}$ for $m \geq 6$.

In order to obtain the corresponding result in $R^{n}$ for $n \geq 4$ we merely supplement $\delta_{\lambda}$ to $\mathcal{S}_{\lambda}^{n}$ by the additional equations $\dot{w}^{i}=-w^{i}, i=4,5, \cdots, n$. For the system $\mathcal{S}_{\lambda}^{n}$ the only compact minimal surfaces are the tori $T_{r}$ in the 3-space $w=0$. Each $T_{r}$ is the (singular chain) boundary of a unique 3-dimensional solid torus $A_{r}$ which is an invariant set under the flow of $\delta_{\lambda}^{n}$. Moreover, $S_{0}$ is characterized as the intersection of all such $A_{r}$. Then the proof that inequivalent values of the irrational $1 / 2 \lambda$ yields topologically inequivalent systems $\int_{\lambda}^{n}$ in $R^{n}$ proceeds just as before, upon ignoring the $w$-coordinates. Therefore $B^{n}(6)=\boldsymbol{\aleph}_{1}$ for $n \geq 4$.

We do not know whether an analogous construction exists for completely unstable differential systems in $R^{3}$, see [8, p. 404] for definitions of this concept and interesting examples.

2. Summary of the theory of separatrices and topological equivalence for planar differential systems. Consider the plane differential system

$$
\dot{x}=f(x, y), \quad \dot{y}=g(x, y),
$$


with $f(x, y)$ and $g(x, y)$ in $C^{1}$ in $R^{2}$, and with no critical points, that is, $f(x, y)^{2}+g(x, y)^{2}>0$ everywhere. Then there are no periodic orbits of $\mathcal{S}$ in $R^{2}$. Each solution curve is a topological image of a line $R$ approaching infinity with both ends. Thus each solution (plus the point at $\infty$ on the sphere $S^{2}$ treated as the one-point compactification of $R^{2}$ ), forms a simple Jordan curve on $S^{2}$. Hence each solution of $\mathcal{S}$ separates $R^{2}$ into two regions (open connected sets), for each of which it is the boundary. If we temporarily ignore the time-sense along the solution of $\mathcal{S}$ then the totality of solution curves is called a regular curve family or foliation ([4], [9]) of the plane, and conversely every (smooth) foliation can be parametrized to recover a differential system. In this section we shall study the foliations defined by differential systems $\mathcal{S}$, always assumed noncritical and $C^{1}$ in $R^{2}$, and we ignore the sense of the solution curves unless otherwise stipulated.

If $S_{1}, S_{2}, S_{3}$ are three distinct solution curves of $\mathcal{S}$ we say that $S_{2}$ separates $S_{1}$ and $S_{3}$, denoted $S_{1}\left|S_{2}\right| S_{3}$ or $S_{3}\left|S_{2}\right| S_{1}$, in case $S_{1}$ and $S_{3}$ lie in different components of $R^{2}-S_{2}$. If none of these three solutions separates the other two, we state that $S_{1}, S_{2}, S_{3}$ form a cyclic triple, denoted $\left|S_{1} S_{2} S_{3}\right|$ without significance to the cyclic order. A collection of solutions for which each triple is cyclic is called a cyclic set.

Kaplan [4] has studied planar regular curve families, such as that determined by the unsensed solutions of $\mathcal{S}$. We refer to [4], [6] for the basic topology of a regular curve family. For instance, a cyclic set is finite or countable and forms the boundary of a simply-connected region. For a countable cyclic set the curves approach infinity uniformly, that is, only a finite number of the curves can meet any given compact set in $R^{2}$.

When $\mathcal{S}$ is pictured in the open unit disc (which is diffeomorphic to $R^{2}$ ) each curve of $\mathcal{S}$ approaches the unit circle (not necessarily with a limiting point) with both ends. Hence the images of the curves of $\mathcal{S}$ in the disc still satisfy the separation properties corresponding to $S_{1}\left|S_{2}\right| S_{3}$ and $\left|S_{1} S_{2} S_{3}\right|$. Since chords of the circle also satisfy similar properties, Kaplan refered to a regular curve family $\mathcal{S}$, when emphasizing the relations $S_{1}\left|S_{2}\right| S_{3}$ and $\left|S_{1} S_{2} S_{3}\right|$, as a chordal system. In fact, using standard methods of plane topology we can map $R^{2}$ topologically onto the disc so that any chosen finite set of curves of $\mathcal{S}$ are carried onto chords of the disc. Thus, when we discuss any finite set of solutions of $\mathcal{S}$ the topology is particularly elementary. For instance two curves $S_{1}$ and $S_{2}$ of $\mathcal{S}$ bound a plane region, with closure $\left[S_{1} S_{2}\right]$ homeomorphic to a strip $\{0 \leq x \leq 1,-\infty<y<\infty\}$ in $R^{2}$. Also any finite cyclic set of solutions of $\mathcal{S}$ bound a simply connected region with a finite number of ends at infinity.

Using the solutions of $\mathcal{S}$ we can define the orbit space $R^{2} / \mathcal{S}$ as the quotient or identification space formed from $R^{2}$ by the equivalence relation of points being on the same solution of $\mathcal{S}$. The orbit space $R^{2} / \mathcal{S}$ bears the usual quotient topology, 
defined as the strongest topology for which the projection map of $R^{2}$ onto $R^{2} / \mathcal{S}$ is continuous. Since the saturation of an open plane set by $\mathcal{S}$ is open in $R^{2}$, the projection is an open map. The orbit space $R^{2} / \mathcal{S}$ is connected, has a countable base, and is a $T$,-space, see [7].

Yet $R^{\frac{1}{2}} / \mathcal{S}$ may not be a Hausdorff separated, or $T_{2}$-space, as the examples in the above section illustrate. If two solutions $S$ and $\bar{S}$ cannot be separated by open sets in $R^{2} / \mathcal{S}$ then each pair of points $P \in S$ and $\bar{P} \in \bar{S}$ have arbitrarily small neighborhoods in $R^{2}$ that are joined by some other solution $\hat{S}$ of $\mathcal{S}$ which lies in $[\bar{S} \bar{S}$. Since $S|\hat{S}| \bar{S}$ is false, we must have $|S \hat{S} \bar{S}|$ as a cyclic triple. Thus we expect a close relation between non-Hausdorff elements of $R^{2} / \mathcal{S}$ and cyclic triples of solutions of $\mathcal{S}$. The next definition and the following remarks and theorem clarify these concepts.

Definition. Consider a noncritical differential system $\mathcal{S}$ in $R^{2}$ with orbit space $R^{2} / \mathcal{S}$. Two solution curves $S_{1}$ and $S_{2}$ of $\mathcal{S}$ are inseparable in $R^{2} / \mathcal{S}$ in case any two open neighborhoods of $S_{1}$ and $S_{2}$ in $R^{2} / \mathcal{S}$ must meet. A solution curve $S$ of $\mathcal{S}$ is a separatrix in case $S$ lies in the closure of the set of inseparable elements of $R^{2} / \mathcal{S}$. We sometimes refer to an inseparable element of $R^{2} / \mathcal{S}$ as an ordinary separatrix and the other separatrices as limit separatrices.

Remark. Let $S_{k}$ be a sequence of ordinary separatrices of $\mathcal{S}$ in $R^{2}$ and assume

$$
\lim _{k \rightarrow \infty} S_{k}=\hat{S}, \text { pointwise in } R^{2},
$$

that is, each point $\hat{P}$ of the solution $\hat{S}$ is the limit in $R^{2}$ of points $P_{k} \in S_{k}$. Then $\hat{S}$ is a separatrix, which might not be an ordinary separatrix. On the other hand, let $\hat{S}$ be a limit separatrix, so each neighborhood of $\hat{S}$ in $R^{2} / \mathcal{S}$ contains ordinary separatrices. Then in $R^{2}$ we can find a sequence of ordinary separatrices $S_{k}$ of $\mathcal{S}$ for which $\lim _{k \rightarrow \infty} S_{k}=\hat{S}$ pointwise in $R^{2}$.

Remark. Consider a completely unstable differential system $\mathcal{S}$ in $R^{n}$, that is, each point $x_{0}$ of $R^{n}$ is wandering [8]. It is reasonable to expect that if $\mathcal{S}$ has no separatrices, then $\mathcal{S}$ is topologically equivalent to the parallel differential system $\dot{x}^{1}=1, \dot{x}^{2}=0, \dot{x}^{3}=0, \cdots, \dot{x}^{n}=0$ in $R^{n}$. In [7] this assertion is proven in $R^{2}$ and $R^{3}$ and shown to be false in $R^{4}$.

In order to prove the equivalence of the above definition of separatrix (which is applicable to very general types of dynamical systems) to one introduced earlier by the author [6], and to unify several uses of this concept, we present a result in plane topology. The next theorem gives two geometrical characterizations of separatrices in terms of the separation and cyclic relations on triples of curves. It is these properties of separatrices that have been used, in various forms ([2], [4], [6]) in the theory of topological equivalence of planar foliations. They will be needed in our classification below.

Theorem 1. Let $\mathcal{S}$ be a noncritical differential system in $R^{2}$. A solution $S \in \mathcal{S}$ 
is a separatrix if and only if

for each pair of solutions $S_{1}$ and $S_{2}$ with $S_{1}|S| S_{2}$ the closed region $\left[S_{1} S_{2}\right]$ contains a cyclic triple of solutions.

On the other band $S \in \mathcal{S}$ is not a separatrix if and only if

there exist solutions $S_{1}$ and $S_{2}$ with. $S_{1}|S| S_{2}$ such that the closed region $\left[S_{1} S_{2}\right]$ is the topological image of the plane strip $\{0 \leq x \leq 1,-\infty<y<\infty\}$ with the curves of $\mathcal{S}$ corresponding to the vertical lines of the strip.

Proof. (1) Assume $S$ is a separatrix and $S_{1}|S| S_{2}$, and we seek a cyclic triple in the closed region $\left[S_{1} S_{2}\right]$. We can assume that $S$ is an ordinary separatrix, since a limit separatrix can be closely approached by ordinary separatrices. Thus there exists a solution $\bar{S}$ that cannot be separated from $S$ in the orbit space $R^{2} / \mathcal{S}$. It is clear that $\bar{S}$ lies in $\left[s_{1} s_{2}\right]$.

The plane closed region $\left[S_{1} S_{2}\right]$ has two ends at infinity. We can suppose that both $S$ and $\bar{S}$ enter and leave from opposite ends, for otherwise $\left|S_{1} \overline{S S}_{2}\right|$ is the required cyclic triple. Since $S$ and $\bar{S}$ are inseparable, there is a solution $\hat{S}$ that lies in the region between $S$ and $\bar{S}$, and $\hat{S}$ approaches very near to points on $S$ and $\bar{S}$. But $\hat{S}$ does not join the two ends of $[\bar{S} \bar{S}]$ because then it would define a separation of $S$ and $\bar{S}$ in $R^{2} / \mathcal{S}$. Thus $|S \hat{S} \bar{S}|$ is the required cyclic triple of solutions in $\left[s_{1} s_{2}\right]$.

(2) Conversely, assume that every pair $S_{1}, S_{2}$ of solutions, with $S_{1}|S| S_{2}$, forms a closed region $\left[S_{1} S_{2}\right]$ containing a cyclic triple, and we shall conclude that $S$ is a separatrix. Take a short segment $L$ transversal to $\mathcal{S}$ across $S$ and let the ends of $L$ lie on neighboring solutions $S_{1}$ and $S_{2}$ so that $\left[S_{1} S_{2}\right]$ contains $S$ in its interior. Then a solution $\widetilde{S}$ crosses the interior of $L$ if and only if $S_{1}|\tilde{S}| S_{2}$, that is, $\tilde{S}$ joins the two ends of $\left[S_{1} S_{2}\right]$. We can linearly order the solutions meeting $L$ by $\tilde{S}^{\prime}>\widetilde{S}$ in case $\tilde{S}^{\prime}|\tilde{S}| S_{1}$, and this corresponds to the order prescribed by the intercepts of $\tilde{S}^{\prime}$ and $\tilde{S}$ along $L$.

Since $\left[S_{1} S_{2}\right]$ contains a cyclic triple, some solution $\hat{S}$ in the interior of this region does not cross $L$ and so $\hat{S}$ must lie at one end of the region $\left[S_{1} S_{2}\right]$. Then $\left|S_{1} \hat{S} S_{2}\right|$. Since $S$ joins the ends of $\left[S_{1} S_{2}\right]$ we must have $\hat{S}$ within $\left[S S_{1}\right]$ or $\left[S_{2} S\right]$. For definiteness we assume $\hat{S}$ lies in $\left[S S_{1}\right]$ and so $\left|S \hat{S} S_{1}\right|$. Now we proceed to advance from $S_{1}$ along $L$ until an ordinary separatrix of $\mathcal{S}$ is obtained in [SS $]$. Use the linear order of solutions $\tilde{S}$ that meet $L$ and let $\tilde{S}_{1}$ be the least upper bound of all such $\tilde{S}$ for which $\hat{S}|\tilde{S}| S_{1}$. We include the possibilities that $\tilde{S}_{1}$ is either $S_{1}$ or $S$, but we claim that $\tilde{S}_{1}$ is an ordinary separatrix curve.

If $\hat{S}\left|\tilde{S}_{1}\right| S_{1}$ or $\tilde{S}_{1}=S_{1}$, then consider the region $\widetilde{R}$ defined as the union of all open regions lying on the $S$-side of all curves $\tilde{S}>\tilde{S}_{1}$. Then $\widetilde{R}$ is a simplyconnected plane region whose boundary is a cyclic set of solutions, including the solution $\widetilde{S}_{1}$ and other solutions that fail to meet $L$. Since $\tilde{S}_{1}$ is inseparable from 
the other components of the boundary of $\widetilde{R}$, we conclude that $\widetilde{S}_{1}$ is an ordinary separatrix.

If $\tilde{S}_{1}$ does not separate $\hat{S}$ and $S_{1}$ or if $\tilde{S}_{1}=S$, then let $\widetilde{R}$ be the union of all open regions lying on the $S_{1}$-side of all curves $\tilde{S}<\tilde{S}_{1}$. Again $\tilde{S}_{1}$ is inseparable from other boundary curves of $\widetilde{R}$ and so $\widetilde{S}_{1}$ is a separatrix. In any case $\left[S_{1} S_{2}\right]$ contains a separatrix that intersects $L$. By shrinking the length of $L$ we obtain a sequence of separatrices that approach $S$. Hence $S$ is also a separatrix.

(3) Next assume that $S$ is not a separatrix and we seek solutions $S_{1}$ and $S_{2}$ such that $S_{1}|S| S_{2}$ and $\left[S_{1} S_{2}\right]$ is a topological image of the standard strip $\{0 \leq x \leq 1,-\infty<y<\infty\}$ in $R^{2}$. By argument (2) above there exist $S_{1}|S| S_{2}$ with $\left[S_{1} S_{2}\right]$ containing no cyclic triple of solutions. Choose a short transversal $L$ across $\mathcal{S}$ and take $S_{1}$ and $S_{2}$ (nearer $S$ if necessary) so that $L$ meets all solutions of $\mathcal{S}$ in $\left[S_{1} S_{2}\right]$. Using the obvious coordinates along $L$ and along the curves of $\mathcal{S}$, we define a one-to-one continuous map of the strip $\{0 \leq x \leq 1,-\infty<y<\infty\}$ onto $\left[S_{1} S_{2}\right]$, with the vertical lines corresponding to the solutions of $\mathcal{S}$. But this map is topological since there are no recurrent motions for a noncritical differential system $\mathcal{S}$ in $R^{2}$.

(4) Finally assume that there exist solutions $S_{1}$ and $S_{2}$ such that $S_{1}|S| S_{2}$ and $\mathcal{S}$ in $\left[S_{1} S_{2}\right]$ is a topological image of the standard strip. We shall show that $S$ is not a separatrix. It is clear that $S$, and each of its neighbors in the strip $\left[S_{1} S_{2}\right]$, lies interior to an open strip of curves that can be separated from one another in the orbit space $R^{2} / \mathcal{S}$, and also separated from any curve outside $\left[S_{1} S_{2}\right]$. Thus $S$, and each of its neighbors, fails to be an ordinary separatrix. Therefore, $S$ is not a separatrix in $\mathcal{S}$. Q.E.D.

Definition. Let $\mathcal{S}$ be a noncritical differential system in $R^{2}$ and let $\Sigma$ be the set of all separatrices of $\mathcal{S}$. Then $\Sigma$ is a closed set in $R^{2}$ and the components of $R^{2}-\Sigma$ will be called the canonical regions of $\mathcal{S}$.

The boundary of each canonical region is a cyclic set (empty, finite, or countable) of separatrix curves. Hence each canonical region is a simply-connected plane region.

As an immediate deduction from Theorem 1, using the same techniques, we assert the following result.

Corollary. Let $R_{0}$ be a canonical region of $\mathcal{S}$ in $R^{2}$. Then $R_{0}$ contains no cyclic triple of solution curves. Moreover, the solutions of $\mathcal{S}$ in $R_{0}$ form a linearly ordered set with each separatrix in the boundary of $R_{0}$ specifying a lower or upper bound. That is, for a separatrix $\bar{S} \in \partial R_{0}$ we define an order, for curves $S_{1}$ and $S_{2}$ of $R_{0}$, by $S_{2}>S_{1}$ in case $S_{2}\left|S_{1}\right| \bar{S}$, which is linear with a lower bound $\bar{S}$.

For details of the proof see [6]. 
We next present two lemmas that will be useful in the classification of polynomial differential systems in the next section of this paper.

Lemma 1. Let $\mathfrak{S}$ be a noncritical differential system in $R^{2}$ and let $S$ be a separatrix which is not the pointwise limit of any sequence $S_{k}$ of distinct ordinary separatrices. Then $S$ is the common boundary of exactly two canonical regions, one on either side of $S$.

Proof. There is an open disc neighborhood of a point $P \in S$ that meets no separatrix, other than $S$. Thus the two open half-discs $D_{1}$ and $D_{2}$ each belong to the complement of the set $\Sigma$ of separatrices of $\mathcal{S}$. Hence $D_{1}$ belongs to a canonical region $R_{1}$ and $D_{2}$ to a different canonical region $R_{2}$. Since the saturation of $D_{1}$ by $\mathcal{S}$ contains no separatrices, $S$ belongs to the boundary of $R_{1}$. Similarly, $S$ belongs to the boundary of $R_{2}$. Moreover any solution curve of $\mathcal{S}$ that is sufficiently close to any prescribed point $Q \in S$ must meet either $D_{1}$ or $D_{2}$. Thus $S$ is the common boundary of exactly two canonical regions. Q.E.D.

Lemma 2. Let $\mathcal{S}$ be a noncritical differential system in $R^{2}$ and assume that $S$ is a separatrix that is the pointwise limit of a sequence of distinct separatrices. Then there exists an infinite cyclic set of solutions of $\mathcal{S}$.

Proof. We can assume that $S_{k}$ is a sequence of distinct ordinary separatrices approaching $S$ pointwise, from one side of $S$. Let $S_{1}$ be so near to $S$ that a transversal segment $L$ joins points on $S$ and $S_{1}$. We can assume $S\left|S_{k+1}\right| S_{k}$ for $k \geq 1$ (using subsequences and appropriate notations), and this ordering agrees with the ordering of the intercepts of $S_{k}$ along $L$. If the closed regions $\left[S_{k+1} S_{k}\right]$ and $\left[S_{k+2} S_{k+1}\right]$ each contain no cyclic triples of solutions, then $\left[S_{k+2} S_{k}\right]$ contains no cyclic triple. But this is impossible, according to Theorem 1 , since $S_{k+1}$ is a separatrix. Thus for each $k \geq 1$ the closed region $\left[S_{k+2} S_{k}\right]$ must contain a solution $\hat{S}_{k}$ such that $\left|S_{k+2} \hat{S}_{k} S_{k}\right|$. The solutions $\hat{S}_{k}$ (ommitting repetitions) form an infinite cyclic set, as required. Q.E.D.

Consider the foliation $\mathcal{S}$ of $R^{2}$ defined by a noncritical differential system. For any subset $\hat{\mathcal{S}}$ of curves of $\mathcal{S}$ we can consider two relations, for distinct triples $s_{1}, S_{2}, s_{3}$ of $\hat{\mathcal{S}}$

$S_{1}\left|S_{2}\right| S_{3}: S_{2}$ separates $S_{1}$ and $S_{3}$ in $R^{2}$

$\left|S_{1} S_{2} S_{3}\right|^{+}: S_{1}, S_{2}, S_{3}$ form a counterclockwise cyclic triple in the order mentioned,

that is, there exists a counterclockwise or positively oriented simple Jordan curve $C$ in the closed region bounded by $S_{1}, S_{2}, S_{3}$ and $C$ touches each of the boundary curves in just a single point $P_{i} \in S_{i}$ with the three points $P_{1}, P_{2}, P_{3}$ in the positive orientation on $C$. Of course, $\left|S_{1} S_{2} S_{3}\right|^{+}$implies that $\left|S_{1} S_{2} S_{3}\right|$. Also $\left|S_{1} S_{2} S_{3}\right|$ implies the validity of exactly one of the possibilities $\left|S_{1} S_{2} S_{3}\right|^{+}$or $\left|S_{2} S_{1} S_{3}\right|^{+}$. 
Definition. Consider a foliation $\delta$ of $R^{2}$ defined by the solutions of a noncritical differential system. A subset $\hat{\varepsilon}$ of curves of $\hat{\varepsilon}$, together with the two relations $S_{1}\left|S_{2}\right| S_{3}$ and $\left|S_{1} S_{2} S_{3}\right|^{+}$among distinct triples of curves $S_{1}, S_{2}, S_{3}$ of $\hat{\mathcal{S}}$ is called a chordal system [4]. The relations on triples of curves are induced naturally by the topology of the oriented plane.

Let $\delta_{1}$ and $\delta_{2}$ be two such foliations of $R^{2}$ with subsets $\hat{\mathcal{S}}_{1}$ and $\hat{\mathcal{S}}_{2}$ defining chordal systems. We say that $\hat{\mathcal{E}}_{1}$ is isomorphic to $\hat{\mathcal{S}}_{2}$ in case there exists a oneto-one correspondence $\Psi$ of the elements of $\hat{\mathcal{E}}_{1}$ and $\hat{\mathcal{S}}_{2}$ such that $\Psi$ preserves the two triple relations.

We note that the correspondence $\Psi$ is not required to be specified by any map of the plane. However, if there does exist an orientation preserving homeomorphism $\Phi$ of $R^{2}$ onto itself such that $\Phi$ specifies a topological 0 -equivalence of the foliations $\mathcal{S}_{1}$ and $\mathcal{S}_{2}$, then clearly the chordal systems $\mathcal{S}_{1}$ and $\mathcal{S}_{2}$ are isomorphic under the induced correspondence on solution curves. Kaplan [4] has proved the converse: if the chordal systems $\delta_{1}$ and $\delta_{2}$ are isomorphic, then the foliations $\delta_{1}$ and $\delta_{2}$ are topologically 0-equivalent in $R^{2}$. However, we shall not use this result, but instead a corresponding theorem involving the separatrix chordal system, as discussed next.

Let $\mathcal{S}$ be a noncritical differential system in $R^{2}$ and we also let $\mathcal{S}$ denote the foliation by solution curves. The separatrices and canonical regions of the foliation of $\mathcal{S}$ are those of the differential system, since their definitions make no reference to the sense of the solution curves. We shall not use the chordal system $\delta$ but merely the subsystem $\Sigma$ defined by the set of all separatrices of $\delta$.

Definition. Let $\mathcal{S}$ be a foliation of $R^{2}$ arising as the solutions of a noncritical differential system, and let $\Sigma$ be the set of all separatrices of $\mathcal{S}$. Choose one solution $S_{a}$ from each canonical region $R_{a}$. Then the separatrix configuration $S \Sigma$ of $\mathcal{S}$ is the chordal system consisting of $\Sigma$ and all the curves $S_{a^{*}}$

Remark. The separatrix configuration $S \Sigma$ of $\mathcal{S}$ is essentially unique, independent of the choice of the solution $S_{a}$ representing $R_{\alpha}$. That is, if $\left\{S_{a}\right\}$ and $\left\{S_{a}^{\prime}\right\}$ are any two such collections of representing curves, and $S \Sigma$ and $(S \Sigma)^{\prime}$ are the corresponding separatrix configurations, then there is an isomorphism $\Psi$ of the chordal system $S \Sigma$ onto $(S \Sigma)^{\prime}$. Moreover, we can demand that $\Psi$ restricts to the identity isomorphism on $\Sigma$, and $\Psi$ carries $S_{a}$ onto $S_{a}^{\prime}$ for each $R_{a}$, see [6]. The existence of the isomorphism $\Psi$ follows from the linear ordering of the solutions of $\mathcal{S}$ within each canonical region $R_{a}$; see the corollary to Theorem 1 above.

We are now in a position to state the fundamental theorem on the 0-equivalence of foliations in the plane. This result was proved by the author in an earlier paper [6, Theorem 8.1].

Proposition. Let $\mathfrak{S}_{1}$ and $\mathfrak{S}_{2}$ be foliations of $R^{2}$ arising as the solutions of noncritical differential systems. Let $\Sigma_{1}$ and $\Sigma_{2}$ be the separatrices, and $S \Sigma_{1}$ 
and $S \Sigma_{2}$ the separatrix configurations, respectively. Then there exists a topological 0-equivalence $\Phi$ of the foliations $\mathcal{S}_{1}$ and $\mathcal{S}_{2}$ if and only if there exists an isomorphism $\Psi$ of the chordal systems $S \Sigma_{1}$ and $S \Sigma_{2}$ such that $\Psi$ restricts to an isomorphism of $\Sigma_{1}$ and $\Sigma_{2}$.

In the next section we shall show that for a foliation $\mathfrak{S}$ consisting of the solutions of a polynomial differential system in $R^{2}$, the separatrix configuration contains only a finite set of curves. Thus we can classify such polynomial differential systems in an effective way by means of their separatrix configurations.

3. Bounds for planar polynomial differential systems. In this section we consider the class $\mathcal{P}^{2}(m)$ of polynomial differential systems, without critical points in $R^{2}$ and with coefficients of degree at most $m=1,2,3, \ldots$. We shall estimate upper bounds for the number $B^{2}(m)$ of topological equivalence classes. To do this we shall study the regular curve family or foliation of $R^{2}$ defined by such a differential system, and we shall estimate the number $B_{f 0}^{2}(m)$ of all topological 0-equivalence classes of such foliations. Since we deal exclusively with planar systems we shall use the notation $\mathcal{P}(m), B(m)$, and $B_{f 0}(m)$ for the above mathematical objects, upon deleting the dimensional superscript 2 . We recall that

$$
B(m) \leq 2 B_{f 0}(m) \leq 4 B(m) .
$$

and hence an estimate for $B_{f 0}(m)$ is sufficient for our purposes.

Lemma 3. Let $\mathfrak{S}$ be a differential system in $\mathcal{P}(m)$. Then no cyclic set of solutions contains more than $2 m+2$ curves.

Proof. Suppose there were a cyclic set $\left\{S_{1}, S_{2}, \ldots, S_{2 m+3}\right\}$ of solutions of $\mathcal{S}$ and let $R_{0}$ be the plane region these curves bound. Let $P_{0}$ be a point in $R_{0}$, say the origin of coordinates, and take a large circle centered at $P_{0}, x^{2}+y^{2}=c^{2}$, for large radius $c>0$. Then this circle must meet each of the $2 m+3$ regions that are separated from $P_{0}$ by the $2 m+3$ curves $s_{1}, s_{2}, \ldots, s_{2 m+3}$.

Consider the radial velocity $\dot{r}$ along solutions of

$$
\dot{x}=f(x, y), \quad \dot{y}=g(x, y) \text {. }
$$

Here $r^{2}=x^{2}+y^{2}$, so $r \dot{r}=x f(x, y)+y g(x, y)$. Since each of the solutions $S_{1}$, $S_{2}, \cdots, S_{2 m+3}$ penetrates the circle $r=c$, we must have $\dot{r}=0$ somewhere on each of $2 m+3$ disjoint arcs of the circle. Thus $x f(x, y)+y g(x, y)$ must vanish at $2 m+3$ distinct points of the circle $x^{2}+y^{2}=c^{2}$. But $x^{2}+y^{2}=c^{2}$ is a quadratic irreducible curve (in the sense of algebraic geometry), and the polynomial $x f+y g$ of degree $m+1$ vanishes at more than $2(m+1)$ points on the circle. Thus $x f+y g$ vanishes identically on the circle $r=c$. (This follows from the classical 
theorem of Bézout which asserts that two plane curves of algebraic degrees $r$ and $s$ cannot intersect in more than $r s$ points, unless the curves have a common component.) But this implies that $\dot{r}=0$ everywhere on $r=c$, and so $r=c$ is a solution of $\mathcal{S}$. This is impossible since each solution of $\mathcal{S}$ approaches infinity in $R^{2}$.

This contradiction proves that $\mathfrak{S}$ has no cyclic set of more than $2(m+1)$ solutions. Q.E.D.

Corollary. Let $\mathcal{S}$ be a differential system in $\mathcal{P}(m)$. Then no separatrix of $\mathcal{S}$ is the pointwise limit of a sequence of distinct separatrices. Hence each separatrix of $\mathcal{S}$ is the common boundary of exactly two canonical regions.

Proof. The two lemmas of the preceding section of this paper give the resuit. For if these were a sequence of distinct separatrices converging pointwise in $R^{2}$, then Lemma 2 asserts the existence of an infinite cyclic set of solutions, which is impossible for $\mathcal{S}$ in $\mathscr{P}(m)$. The other conclusion follows from the first of those two lemmas. Q.E.D.

Lemma 3 shows that each canonical region of $\mathcal{S} \in \mathcal{P}(m)$ is bounded by a cyclic set of at most $2 m+2$ separatrices. The next result uses this bound to show that $\mathcal{S}$ has only a finite number of separatrices and canonical regions.

Lemma 4. Let $\mathfrak{S}$ be a differential system in $\mathcal{P}(m)$. Then $\mathfrak{S}$ bas at most $6 m$ separatrices, and at most $6 m+1$ canonical regions.

Proof. Choose a canonical region $R_{0}$ that either has more than two separatrices in its boundary, or else $R_{0}$ is bounded by exactly two separatrices which form a cyclic triple with some representative solution from $R_{0}$. If no such canonical region $R_{0}$ exists, then there are no separatrices of $\mathcal{S}$ and the theorem is trivially true. Thus we assume the existence of $R_{0}$ and fix the origin $P_{0}$ inside $R_{0}$. We select a cyclic set $\sigma_{0}$ of solutions from the closed region $\bar{R}_{0}$, where $\sigma_{0}$ consists of some boundary separatrices and, possibly, a representative solution from $R_{0}$. Further, $\sigma_{0}$ contains at least 3 members, and at most $2 m+2$ members.

We shall enlarge $\bar{R}_{0}$ to a union of closed canonical regions, and with each step of the process we shall simultaneously enlarge the number of members of the cyclic set $\sigma_{0}$.

Take a separatrix $S_{1} \in \sigma_{0}$ from the boundary of $\bar{R}_{0}$. Then elementary geometry of chordal systems shows that exactly one of the following cases must obtain:

(a) the canonical region $R_{1}$ exterior to $S_{1}$ (that is, the region with $S_{1}$ in the boundary, yet $R_{1}$ separated from $P_{0}$ by $S_{1}$ ) has only $S_{1}$ in its boundary;

(b) the canonical region $R_{1}$ exterior to $S_{1}$ has 3 or more separatrices in its boundary;

(c) the canonical region $R_{1}$ exterior to $S_{1}$ is bounded by exactly 2 separatrices which form a cyclic triple with a representative solution from $R_{1}$; 
(d) the canonical region $R_{1}$ exterior to $S_{1}$ is bounded by exactly 2 separatrices $S_{1}$ and $S_{1}^{\prime}$ and there is no cyclic set in $\bar{R}_{1}$; but there exists a canonical region $R_{1}^{\prime}$ exterior to $S_{1}^{\prime}$ such that $\bar{R}_{1}^{\prime}$ contains a cyclic triple of solutions.

In cases (b) and (c) we enlarge $\bar{R}_{0}$ to the union $\bar{R}_{0} \cup \bar{R}_{1}$ and in case (d) we obtain $\bar{R}_{0} \cup \bar{R}_{1} \cup \bar{R}_{1}^{\prime}$. We also replace the cyclic set $\sigma_{0}$ by $\sigma_{1}$ which consists of all the curves of $\sigma_{0}$, excluding $S_{1}$, and with 2 additional curves selected from the closed region $\bar{R}_{1}$ (or from the boundary of $\bar{R}_{1}^{\prime}$ and a representing curve from $R_{1}^{\prime}$ in case (d)). Provided the separatrix $S_{1}$ is not of type (a), we can enlarge the closed region $\bar{R}_{0}$ to a finite union of closed canonical regions, and we enlarge the size of the cyclic set $\sigma_{0}$ to another cyclic set $\sigma_{1}$ which contains one more member.

We can repeat this process of enlargement by expanding outward across the separatrices that form the outer boundary of the closed region already obtained. For each step of enlargement we add at most 2 canonical regions, but we also increase the size of the corresponding cyclic set by one member. At each step the enlarged closed region is simply-connected and is bounded by a finite cyclic set of separatrices. The process of enlargement terminates only if every one of these exterior bounding separatrices is of type (a) above.

Since no cyclic set can have $2 m+3$ elements, the process of enlargement can proceed for at most $(2 m+2)-3=2 m-1$ steps. Thus the total number of canonical regions in the final closed region is at most $1+2(2 m-1)=4 m-1$. In addition there is just one canonical region exterior to each of the separatrices forming the cyclic boundary of this closed region, that is, there are at most $2 m+2$ further exterior canonical regions. Thus the total number of canonical regions of $\delta$ does not exceed

$$
(4 m-1)+(2 m+2)=6 m+1 .
$$

Since the boundary of each canonical region consists of a cyclic set of at most $2 m+2$ separatrices, and since every separatrix belongs to such a boundary, there are at most $(6 m+1)(2 m+2)$ separatrices of $\mathcal{S}$. However we can make a more accurate count for the number of separatrices.

Pick a separatrix $\hat{S}$ such that all other separatrices of $\mathfrak{d}$ lie on just one side of $\hat{S}$. Then adjacent to $\hat{S}$ on the side containing all other separatrices is a canonical region $\hat{R}$. Further, for each separatrix $\tilde{S}$ of $\mathfrak{S}$ there is just one canonical region $\widetilde{R}$ adjacent to $\tilde{S}$ on the side that $\tilde{S}$ separates from $\hat{S}$. Moreover, every canonical region, excepting the one whose single boundary curve is $\hat{S}$, is adjacent to one such separatrix. Thus the number of canonical regions of $\mathcal{S}$ exceeds the number of separatrices by one. Hence the number of separatrices of $\mathcal{S}$ is at most 6m. Q.E.D.

Theorem 2. Let $B(m)$ be the number of topological equivalence classes of 
$\mathcal{P}(m)$, the set of planar noncritical differential systems with polynomial coefficients of degree not exceeding $m$. Then

$$
B(m) \leq 2^{18 m-2}(m+1)^{24 m-5} \text { for } m=1,2,3, \cdots
$$

Proof. We shall first estimate $B_{f 0}(\mathrm{~m})$, the number of topological 0-equivalence classes of foliations defined by differential systems $\mathcal{S} \in \mathcal{P}(m)$. Each such 0-equivalence class of foliations is characterized by the isomorphism class of the corresponding separatrix configuration $S \Sigma$. Thus we proceed to compute an upper bound for the possible isomorphism types of separatrix configurations for systems $\mathcal{S} \in \mathcal{P}(m)$.

Consider any foliation for a system $\mathfrak{S} \in \mathcal{P}(m)$. We call a separatrix $S$ of $\mathcal{S}$ an exterior separatrix in case $S$ does not separate any two other separatrices of $S$ in $R^{2}$. The collection of all exterior separatrices of $\mathcal{S}$ is the exterior cycle $\sigma$. Then $\sigma$ is a cyclic set of $p$ members, where $2 \leq p \leq 2 m+2$ (unless there are no separatrices, which implies that $\delta$ is 0 -equivalent to parallel lines filling $R^{2}$ ). The isomorphism class of $\Sigma$ can be described by the positions of the ends of the curves of $\Sigma$ relative to the members of $\sigma$. We proceed by this approach.

Fix an integer $p$ in $2 \leq p \leq 2 m+2$, and let $\sigma$ be a cyclic set of $p$ chords in the unit disc. We shall estimate an upper bound for the number of isomorphism types that can be realized as chordal systems $S \Sigma$ for this exterior cyclic set $\sigma$ of some $\mathfrak{S} \in \mathcal{P}(m)$. Now $S \Sigma$ contains at most $6 m$ separatrices, and $6 m+1$ repre-

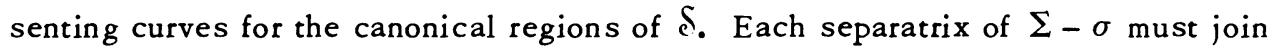
two different ends of the region bounded by the chords of $\sigma$, for otherwise $\sigma$ would fail to contain all exterior separatrices of $\mathcal{S}$. There are $p$ ends for the region bounded by $\sigma$ and there are at most $6 m-p$ separatrices of $\Sigma-\sigma$. Each of the se other separatrices is described by the positions of its ends, relative to the ends of $\sigma$. Also each such separatrix in $\Sigma-\sigma$ must join two different ends of $\sigma$, since it is not an exterior separatrix. Thus there are at most $(\underset{2}{p})$ possible positions for each of the separatrices of $\Sigma-\sigma$, and hence at most $[p(p-1) / 2]^{6 m-p}$ isomorphism classes for $\Sigma$. Many of these assignments for the positions of $\Sigma$ are impossible, or else yield isomorphic chordal systems. But isomorphic separatrix chordal systems $\Sigma_{1}$ and $\Sigma_{2}$ for systems $\mathcal{S}_{1}$ and $\mathcal{S}_{2} \in \mathscr{P}(m)$, having exterior $p$-cycles, will be counted by the same positional assignment of the ends of the $6 m-p$ separatrices. Also each positional assignment defines at most one isomorphism class for $\Sigma$.

Next for each such chordal system $\Sigma$ we proceed to assign positions for the representative curves, so as to count the possibilities for isomorphism classes of $S \Sigma$. Of course $p$ of the representative curves are parallel and just outside the separatrices of $\sigma$. Then, at most $6 m+1-p$ representative curves must each be specified by the position of its two ends within the, at most, $2 m+2$ ends of its 
canonical region. Therefore, the number of isomorphism classes of $S \Sigma$, for a fixed $\Sigma$, is at most $(2 m+2)^{2(6 m+1-p)}$.

Thus the isomorphism classes for separatrix configurations $S \Sigma$ of $e_{\epsilon} \in \mathcal{P}(m)$, with exterior $p$-cycle, do not exceed the product $[p(p-1) / 2]^{6 m-p}(2 m+2)^{2(6 m+1-p)}$. Hence, we obtain the upper bound

$$
B_{f 0}(m) \leq \sum_{p=2}^{2 m+2}\left[\frac{p(p-1)}{2}\right]^{6 m-p}(2 m+2)^{2(6 m+1-p)} .
$$

If we raise the estimate in order to simplify the expression, we obtain

$$
B_{f 0}(m) \leq(2 m+2)^{12 m-2} \sum_{p=2}^{2 m+2}\left(\frac{p^{2}}{2}\right)^{6 m-2} \leq(2 m+2)^{12 m-2} \frac{(2 m+2)^{12 m-4}}{2^{6 m-2}}(2 m+2) .
$$

Thus

$$
B_{f 0}(m) \leq(2 m+2)^{24 m-5} / 2^{6 m-2}
$$

and

$$
B(m) \leq(2 m+2)^{24 m-5} / 2^{6 m-3} \leq 2^{18 m-2}(m+1)^{24 m-5},
$$

as stated. Q.E.D.

Corollary. The asymptotic behavior of $B(m)$ is

$$
\log \log B(m) \sim \log m,
$$

that is,

$$
\lim _{m \rightarrow \infty} \frac{\log \log B(m)}{\log m}=1 .
$$

Proof. From our earlier estimates (see the note following Example 2 of $\$ 1$ above),

$$
2^{m / 2} \leq B(m) \leq 2^{18 m-2}(m+1)^{24 m-5} \leq m^{25 m} \text { for large } m .
$$

Then (using natural logarithims)

$$
m \log \sqrt{2} \leq \log B(m) \leq 25 m \log m,
$$

and

$$
\log m+\log \log \sqrt{2} \leq \log \log B(m) \leq \log m+\log 25+\log \log m .
$$

Hence (independent of the base of logarithims),

$$
\lim _{m \rightarrow \infty} \frac{\log \log B(m)}{\log m}=1
$$

as required. Q.E.D. 


\section{BIBLIOGRAPHY}

1. A. A. Andronov, E. A. Leontovič, I. I. Gordon and A. G. Măer, Qualitative theory of dynamic systems of the second order, "Nauka", Moscow, 1966. (Russian) MR 33 $\# 7650$.

2. A. Beck, Plane flows with few stagnation points, Bull. Amer. Math. Soc. 71(1965), 886-890. MR $32 \# 441$.

3. G. Hardy and E. Wright, An introduction to the theory of numbers, Clarendon Press, Oxford, 1938.

4. W. Kaplan, Regular curve-families filling the plane. I, II, Duke Math. J. 7 (1940), 154-185; ibid. 8 (1941), 11-46. MR 2, 322.

5. J. Majerczyk, Separatrices of plane dynamical systems, Dokl. Akad. Nauk SSSR 140 (1961), 1008-1010 = Soviet Math. Dokl. 2(1961), 1301-1303. MR 24 \#A1460.

6. L. Markus, Global structure of ordinary differential equations in the plane, Trans. Amer. Math. Soc. 76 (1954), 127-148. MR 15, 704.

7. - Parallel dynamical systems, Topology 8 (1968), 47-57.' MR 38 \#2806.

8. V. V. Nemyckii and V. V. Stepanov, Qualitative theory of differential equations, OGIZ, Moscow, 1947; English transl., Princeton Math. Series, no. 22, Princeton Univ. Press, Princeton, N. J., 1960. MR $22 \# 12258$.

9. H. Whitney, Regular families of curves, Ann. of Math. (2) 34 (1933), 244-270.

DEP ARTMENT OF M ATHEMATICS, UNIVERSTY OF MINNESOTA, MINNEAPOLIS, MINNESOTA 55455

DEPARTMENT OF MATHEMATICS, UNIVERSITY OF WARWICK, COVENTRY, ENGLAND 